\title{
Dual role of TGFBR3 in bladder cancer
}

\author{
XIAO-LONG LIU ${ }^{1 *}$, KEBING XIAO $^{1 *}$, BOXIN XUE $^{1}$, DONGRONG YANG ${ }^{1}$, \\ ZHE LEI ${ }^{2,3}$, YUXI SHAN ${ }^{1}$ and HONG-TAO ZHANG ${ }^{2,3}$ \\ ${ }^{1}$ Department of Urology, The Second Affiliated Hospital of Soochow University, Suzhou, Jiangsu 215004; \\ ${ }^{2}$ Soochow University Laboratory of Cancer Molecular Genetics, Medical College of Soochow University, Suzhou, \\ Jiangsu 215123; ${ }^{3}$ Suzhou Key Laboratory for Molecular Cancer Genetics, Suzhou, Jiangsu 215123, P.R. China
}

Received April 16, 2013; Accepted June 12, 2013

DOI: 10.3892/or.2013.2599

\begin{abstract}
Bladder cancer is one of the most common genitourinary malignant diseases worldwide. More than $90 \%$ of bladder cancer cases are bladder urothelial carcinoma (BUC). Although transforming growth factor- $\beta$ III receptor (TGFBR3) has been suggested to play a dual role in cancer progression, little is known about TGFBR 3 in bladder cancer. In the present study, fresh tumor and the corresponding paracarcinoma tissue specimens were collected from 56 bladder urothelial carcinoma patients. TGFBR 3 expression in these tissues was determined by western blotting. TGFBR3 was also detected in the human normal urothelial cell line SV-HUC-1, the human superficial urothelial bladder cancer cell line 5637, and the human invasive bladder cancer cell line T24 using western blotting and quantitative PCR. Cell growth, motility and invasion were also analyzed in the control and the TGFBR 3 gene-silenced T2 4 cells. As a result, the expression of TGFBR3 was reduced (18/30) in most superficial bladder urothelial carcinoma tissues compared to the corresponding normal tissues, whereas TGFBR3 expression was more enhanced (19/26) in the invasive samples. Similarly, an increase of TGFBR 3 expression was found in T24 cells, but a decrease was observed in 5637 cells. Knockdown of TGFBR3 in T24 cells resulted in decreased cell growth, motility and invasion. In conclusion, these findings suggest that TGFBR3 may play a dichotomous role in human bladder cancer, acting as both a tumor suppressor and as a tumor promoter.
\end{abstract}

Correspondence to: Dr Yuxi Shan, Department of Urology, The Second Affiliated Hospital of Soochow University, 1055 Sanxiang Road, Suzhou, Jiangsu 215004, P.R. China

E-mail: shanyuxi1002@126.com

Professor Hong-Tao Zhang, Soochow University Laboratory of Cancer Molecular Genetics, Medical College of Soochow University, 199 Ren'ai Road, Sino-Singapore Industrial Park, Suzhou, Jiangsu 215123, P.R. China

E-mail: htzhang@suda.edu.cn

${ }^{*}$ Contributed equally

Key words: TGFBR3, bladder urothelial carcinoma, progression, siRNA

\section{Introduction}

Bladder cancer is one of the most common genitourinary malignant diseases worldwide. More than $90 \%$ of bladder cancer cases are bladder urothelial carcinoma (BUC) (1). At the time of diagnosis, $75-85 \%$ of the patients harbored superficial bladder cancer (non-muscle-invasive bladder cancer; NMIBC) (2). Despite transurethral resection of bladder tumor (TURBT) and intravesical therapy, $1-45 \%$ of cases progress to invasive bladder cancer (muscle-invasive bladder cancer; MIBC) within 5 years (3). Approximately 50\% patients with MIBC develop metastatic disease, which is almost invariably lethal (4). The exact mechanisms of tumor formation and progression are not yet completely understood. Genetic and molecular factors may both play a role in the process (5).

Transforming growth factor- $\beta$ (TGF- $\beta$ ) superfamily signaling pathways play opposing roles in human cancer, functioning both as tumor suppressors and as tumor promoters $(6,7)$. TGF- $\beta$ superfamily ligands exert these biological effects by directly or indirectly binding to three cell surface receptors, type I (TGFBR1), type II (TGFBR2) and type III (TGFBR3) transforming growth factor- $\beta$ receptor. Ligands binding to TGFBR2 cause TGFBR2 to phosphorylate TGFBR1; the activated TGFBR1 phosphorylates and in turn activates Smad transcription factors, which move into the nucleus and regulate target genes by interacting with other transcription factors (8). In the TGF- $\beta$ superfamily signaling pathways, TGFBR 3 , as a co-receptor, plays an important role in mediating liganddependent TGF- $\beta$ superfamily signaling and possesses important ligand-independent functions (7).

Loss or reduced expression of TGFBR3 has been demonstrated in multiple types of human cancer including breast, prostate, ovarian, pancreatic, non-small cell lung cancer and renal cell carcinoma, whereas restoring TGFBR3 expression results in decreased migration and invasion. These results indicate that TGFBR3 may play a suppressive role in cancer progression (9-14). However, Gatza et al (15) reported that TGFBR3 expression was actually enhanced in human colon cancer and may even function to promote colon cancer progression. These data suggest that TGFBR3 may play a dual role in cancer progression. However, little is known about TGFBR3 in bladder cancer. In the present study, we investigated the expression of TGFBR3 in BUC tissues and cells. We also silenced the TGFBR3 gene by chemically synthesizing siRNA 
in bladder cancer cell line T24 and determined the growth, motility and invasion of the cells after the gene knockdown. Our results suggest that TGFBR3 may play diverse roles in bladder cancer, functioning to suppress tumorigenesis in early stages and promoting cancer progression in later stages.

\section{Materials and methods}

Specimens. Fresh tumor and the corresponding paracarcinoma tissue specimens were collected from 56 BUC patients who participated in the study at The Second Affiliated Hospital of Soochow University. Of the 56 patients, 27 had undergone transurethral resection of bladder tumor (TURBT), 25 had undergone radical cystectomy and 4 had undergone partial cystectomy. Tumors were pathologically staged according to the 2010 American Joint Committee on Cancer TNM staging system, and tumors were graded using the 1973 World Health Organization (WHO) classification (Table I). The present study was approved by The Academic and Ethics Advisory Board of the Second Affiliated Hospital of Soochow University. None of these patients had received radiotherapy or chemotherapy prior to surgery. These specimens were snap-frozen and stored in liquid nitrogen immediately after the operation and until analysis.

Cell culture and transfection. The human bladder cancer cell lines 5637 and T24 and the human normal urothelial cell line SV-HUC-1 were obtained from the Shanghai Cell Bank of the Chinese Academy of Sciences (Shanghai, China). All cell lines were cultured in RPMI-1640 medium supplemented with 10\% fetal bovine serum (FBS), $100 \mathrm{U} / \mathrm{ml}$ of penicillin and $100 \mathrm{mg} / \mathrm{l}$ of streptomycin in a humidified atmosphere of $95 \%$ air maintained at $37^{\circ} \mathrm{C}$. The siRNAs were designed and chemically synthesized by Shanghai GenePharma Co., Ltd. (Shanghai, China). Then, $5 \times 10^{5}$ cells/well were planted in a 6 -well plate. A 30-50\% confluence was achieved after $24 \mathrm{~h}$. Transfections were performed using Entranster-R siRNA transfection reagent (Entranster, China) according to the manufacturer's instructions. Control for non-specific effects caused by the transfection procedure is referred to as mock-transfection control (mock). The sequences of negative control siRNA (siRNA-NC), the sequences of specific TGFBR3 siRNAs and their targeting sites are listed in Table II.

RNA isolation and quantitative RT-PCR (qRT-PCR). RNAiso Plus reagent (Takara Bio Inc., Shiga, Japan) was used to isolate total RNA according to the manufacturer's protocol. RNA $(\sim 150 \mathrm{ng})$ was reverse transcribed in a $20 \mu \mathrm{l}$ reaction according to the manufacturer's instructions. The duplex qRT-PCR was performed in a $25 \mu 1$ reaction system using the ABI PRISM 7500 Real-Time PCR system (Applied Biosystems, Foster City, CA, USA) with SYBR-Green qPCR SuperMix-UDG (Invitrogen, Carlsbad, CA, USA). All Ct values were equilibrated to the GAPDH control. All reactions were performed in triplicate. PCR primers for specific genes and GAPDH are shown in Table III (from PrimerBank http://pga.mgh.harvard. edu/primerbank/).

Western blotting. Cell lysate was prepared by extracting proteins with RIPA buffer (Fermentas, Ontario, Canada)
Table I. Clinical characteristics of the 56 patients who participated in this study.

\begin{tabular}{lrrrr}
\hline & & \multicolumn{2}{c}{ Gender $^{\mathrm{a}}$} \\
\cline { 3 - 4 } $\begin{array}{l}\text { Pathological } \\
\text { parameters }\end{array}$ & Tumors $^{\mathrm{a}}$ & Male & Female & Age (years) \\
\hline pT stage & & & & \\
Ta/T1 & 30 & 24 & 6 & $28-78$ \\
T2 & 16 & 16 & 0 & $42-87$ \\
T3 & 7 & 6 & 1 & $61-72$ \\
T4 & 3 & 2 & 1 & $46-74$ \\
pN stage & & & & \\
N0 & 44 & 39 & 5 & $28-79$ \\
N+ & 12 & 9 & 3 & $64-87$ \\
pM stage & & & & \\
M0 & 56 & 48 & 8 & $28-87$ \\
M1 & 0 & 0 & 0 & - \\
Grade & & & & \\
1 & 25 & 21 & 4 & $33-87$ \\
2 & 20 & 17 & 3 & $28-86$ \\
3 & 11 & 10 & 1 & $46-79$ \\
\hline
\end{tabular}

${ }^{\mathrm{a} N u m b e r}$ of cases.

supplemented with $1 \%$ protease inhibitors (Sigma). The cells or tissues were lysed in boiling sample buffer and resolved by sodium dodecyl sulfate-polyacrylamide gel electrophoresis and immunoblotted for the proteins of interest. TGFBR3 antibody was purchased from R\&D Systems (Minneapolis, MN, USA), and a 1:1,000 dilution was used for immunoblot analysis. Secondary antibody was purchased from Santa Cruz Biotechnology, and a 1:5,000 dilution was used for immunoblot analysis. Membranes were blocked with $0.25 \%$ gelatin in TBST, followed by incubation with primary antibodies overnight at $4^{\circ} \mathrm{C}$. Membranes were washed three times in TBST, followed by incubation with secondary antibody for $2 \mathrm{~h}$ at room temperature. Membranes were washed three times in TBST and developed with enhanced chemiluminescence (Fermentas).

Water-soluble tetrazolium salt (WST-1) assay. The viability of the cells was determined by the WST-1 assay according to the manufacturer's protocol. T24 cells $\left(5 \times 10^{3} /\right.$ well) were seeded into 96-well flat-bottom plates. Twenty four hours later, the cells were transfected with siRNAs. Following transfection for $0,24,48$ and $72 \mathrm{~h}$, cell viability was determined. One and a half hours before termination of the experiment, $10 \mu \mathrm{l}$ of WST (Beyotime Institute of Biotechnology, Jiangsu, China) was added to each $100 \mu 1$ well. Analysis was performed with a microplate reader (Molecular Devices, Sunnyvale, CA, USA) at OD450 and OD620 nm.

Colony formation assay. T24 cells were seeded in 6-well plates. The following day, they were transfected as previously described according to the manufacturer's instructions. Forty-eight hours after transfection, the cells were harvested 
Table II. The sequences of siRNAs and their targeting sites.

\begin{tabular}{llc}
\hline siRNA & \multicolumn{1}{c}{ Sequences } & Targeting sites \\
\hline siRNA-1 & Sense: GGU CACA CUUC ACCU GAAUTT & $761-779$ \\
Antisense: AUU CAGG UGAA GUGU GACCTT & $1298-1316$ \\
siRNA-2 & Sense: GGA UCUU GAAG UGGU CAAATT & $2061-2079$ \\
siRNA-3 & Antisense: UUU GACC ACUU CAAG AUCCTT & $2731-2749$ \\
& Sense: GGU GUGGU CUACU AUAACUTT & No \\
siRNA-NC & Antisense: AGU UAUAG UAGA CCACACCTT & \\
& Sense: GGGCC AUGAUG GAGA AUAATT & \\
& Antisense: UUAUU CUGCAU CAU GGCCCTT & \\
& Sense: UUC UCC GAA CGU GUC ACGUTT & \\
\end{tabular}

Table III. Primers used for qRT-PCR.

\begin{tabular}{llr}
\hline Gene & \multicolumn{1}{c}{ Sequences } & Size of PCR product (bp) \\
\hline TGFBR3 & Sense: TGGGGTCTCCAGACTGTTTTT & 149 \\
& Antisense: CTGCTCCATACTCTTTTCGGG & 101 \\
GAPDH & Sense: ACAACTTTGGTATCGTGGAAGG & \\
& Antisense: GCCATCACGCCACAGTTTC & \\
\hline
\end{tabular}

and plated in a 6 -well plate at a density of 200 cells/well. The cells were cultured in an incubator at $37^{\circ} \mathrm{C}$ for 14 days. On day 15 , cells were washed with PBS, fixed with absolute methanol, and stained with Giemsa. The colonies with $>50$ cells were counted. All assays were repeated at least three times, and the data are presented as the means \pm SD.

Analysis of DNA contents by propidium iodide (PI) staining. PI staining reagent was purchased from Beyotime Institute of Biotechnology. PI staining was performed according to the manufacturer's protocol. Cells $\left(10^{6}\right)$ were briefly fixed with $70 \%$ alcohol (v/v) for at least $24 \mathrm{~h}$. After washing with PBS, the cells were incubated with PI staining reagent at $37^{\circ} \mathrm{C}$ for $30 \mathrm{~min}$. This analysis was performed with FC500 flow cytometry (Beckman Coulter, Brea, CA, USA). Cells with subdiploid DNA content were considered apoptotic cells. Cell cycle distributions were analyzed by the cell fit software package.

Monolayer wound healing. T24 cells $\left(1 \times 10^{5}\right)$ were plated in each well of a 6-well plate. Following overnight incubation, the cells were treated with siRNAs $(50 \mathrm{nM}$ siControl, $50 \mathrm{nM}$ siRNA-TGFBR3) for $48 \mathrm{~h}$. Confluent sheets of cells were wounded by scraping with a pipette tip, rinsed with PBS to remove dislodged cells two times, and added to a fresh medium. At 0 and $24 \mathrm{~h}$ after the scratching, images were captured with an Olympus inverted microscope (Olympus, Tokyo, Japan) at a magnification of $x 40$. Cells were maintained at $37^{\circ} \mathrm{C}$ and $5 \%$ $\mathrm{CO}_{2}$ during incubation.

Transwell motility assay and Matrigel invasion assay. The motility and invasive activity of T24 cells were analyzed in
Table IV. Summary of TGFBR3 expression in patients with bladder cancer.

\begin{tabular}{lcr}
\hline & \multicolumn{2}{c}{ TGFBR3 expression $^{\mathrm{a}}$} \\
\cline { 2 - 2 } Bladder cancer $^{\mathrm{b}}$ & Increased & Decreased \\
\hline MIBC & 19 & 5 \\
NMIBC & 8 & 18
\end{tabular}

${ }^{a}$ TGFBR3 expression in carcinoma tissues compared with those in paracarcinoma tissues. $\chi_{\mathrm{df}=1}^{2}=11.768, \mathrm{P}=0.001$. MIBC, muscle-invasive bladder cancer; NMIBC, non-muscle-invasive bladder cancer.

a transwell cell culture chamber (Costar 3422, polycarbonate membrane, 24 -well format, $8-\mu \mathrm{m}$ pore size; Corning, Inc., New York, NY, USA). T24 cells were plated in each well of a 6-well plate at a density of $8 \times 10^{4}$ cells/well. After overnight incubation, the cells were treated with siRNAs (50 nM siControl, $50 \mathrm{nM}$ siRNA-TGFBR3) for $48 \mathrm{~h}$. T2 4 cells $\left(1 \times 10^{5} /\right.$ well for motility assay, $5 \times 10^{4} /$ well for invasion assay) were then applied to the upper chamber in a $200 \mu \mathrm{l}$ RPMI-1640 medium supplemented with 5\% FBS. RPMI-1640 medium supplemented with $20 \%$ FBS was added to the lower chamber to act as a chemoattractant. Cells were then allowed to seep out of the Matrigel, across the membrane, at $37^{\circ} \mathrm{C}$ for $24 \mathrm{~h}$. Non-invasive cells were then removed from the top compartment of the transwell with a cotton swab. The filters were fixed with methanol and stained with $0.1 \%$ crystal violet. The cells that had invaded 


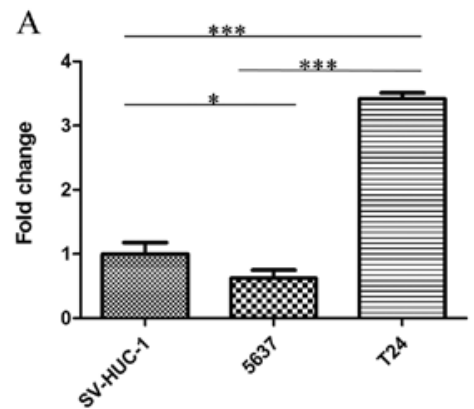

B

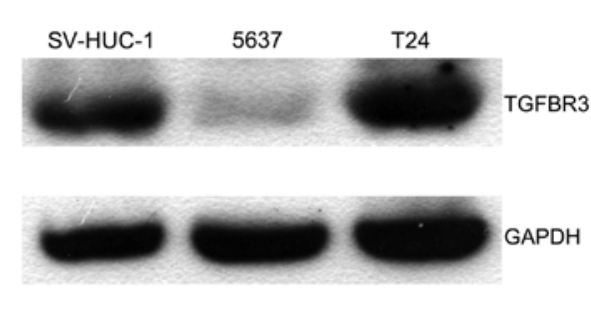

Figure 1. TGFBR3 expression in human bladder cancer cell lines. (A) TGFBR3 mRNA expression was detected by qRT-PCR in the human normal bladder cell line (SV-HUC-1) and the human bladder cancer cell lines (5637 and T24). "P<0.05, ${ }^{* * *} \mathrm{P}<0.001$. Columns, mean of 3 independent experiments; Bars, SD. (B) Western blotting for TGFBR3 protein expression in SV-HUC-1, 5637 and T24 cell lines.

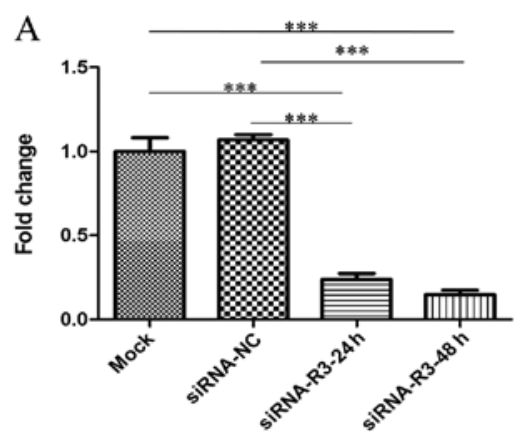

B

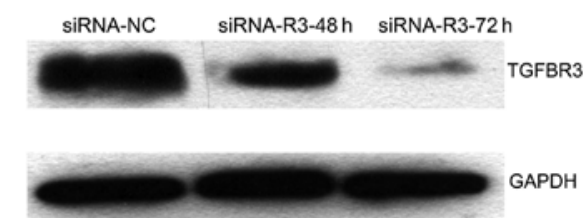

Figure 2. Suppression of TGFBR3 by siRNA in T24 cells. T24 cells were transfected with $50 \mathrm{nM}$ siRNA-TGFBR3 or siRNA-NC. The mRNA (A) or protein (B) expression of TGFBR3 was detected after the treatment. *** $\mathrm{P}<0.001$. Columns, mean of 3 independent experiments; bars, SD.

through Matrigel and filter to the lower surface were manually counted under a microscope in five predetermined fields at a magnification of $x 100$.

Statistical analysis. The chi-square test was used to determine whether increased TGFBR3 expression was associated with muscle-invasion. The one-way analysis of variance (ANOVA) was used to compare statistical significance between treatment groups and controls. The two-tailed equal variance Student's t-test was used to compare the average number of migrated cells to the invaded cells of the five fields. All statistical analyses were performed using SPSS statistical software version 17.0. $\mathrm{P} \leq 0.05$ was considered to indicate a statistically significant difference.

\section{Results}

TGFBR3 expression in human bladder cancer tissues. To explore the possible role of TGFBR3 in bladder tumorigenesis, we detected the protein expression of TGFBR3 in 56 pairs of BUCs and corresponding paracarcinoma tissues using western blotting. TGFBR3 protein expression was significantly altered in 50/56 (89.26\%) tumor samples when compared to paracarcinoma tissues. In 24 altered pairs of MIBC, TGFBR3 was increased in 19 of the tumor samples, and decreased in 5 patients. Of the 26 altered pairs of NMIBC, 8 patients experienced increased TGFBR3 in tumor focuses, and 18 patients showed decreased TGFBR3 in the tumors (Table IV). Thus, it appears that the increased TGFBR3 expression in the bladder cancer tissues was associated with muscle-invasion (MIBC).
TGFBR3 expression in normal bladder epithelium and bladder cancer cell lines. To identify TGFBR3 expression in bladder cancer cells, we detected TGFBR3 expression in normal bladder epithelium cell line SV-HUC-1, superficially derived bladder cell line 5637, and invasive cell line T24 by qRT-PCR via western blotting. We found that both mRNA and protein expression of TGFBR3 was decreased in 5637 cells when compared to SV-HUC-1 cells, whereas the expression of TGFBR3 significantly increased in T24 cells (Fig. 1).

Knockdown of TGFBR3 inhibits cell viability and colony formation, but not cell cycle arrest in T24 cells. In order to achieve efficient and specific TGFBR3 depletion, T24 cells were transfected with $50 \mathrm{nM}$ of siRNA-NC, siRNA-1, siRNA-2, siRNA-3 and siRNA-4, respectively. We found that the expression of TGFBR3 was decreased by up to $80-85 \%$ when transfected with siRNA-1, siRNA-2 and siRNA-3 (data not shown). Thus, we mixed them together equally into a siRNA pool and called it siRNA-TGFBR3. The expression of TGFBR3 in T24 cells that were separately transfected with siRNA-TGFBR3 at various concentrations was detected by qRT-PCR. Consequently, 50 nM siRNA-TGFBR3 achieved an effective knockdown; therefore, $50 \mathrm{nM}$ siRNA-TGFBR3 was used as the final concentration in the following experiments to investigate the effects of silencing TGFBR3 on T24 cells at $48 \mathrm{~h}$ post-transfection.

T2 4 cells were transfected with $50 \mathrm{nM}$ siRNA-TGFBR 3 or siRNA-NC. The TGFBR3 mRNA expression was detected by qRT-PCR at 24 and $48 \mathrm{~h}$ (Fig. 2A) and TGFBR3 protein expression was detected by western blotting at 48 and 72 h (Fig. 2B) 
A

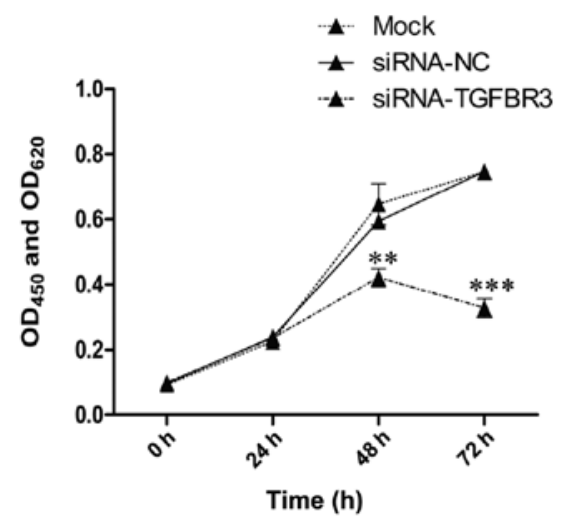

B

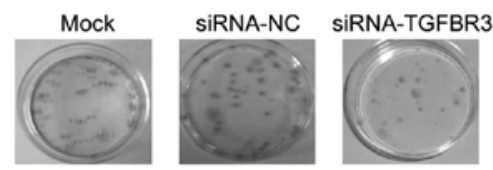

$\mathrm{C}$

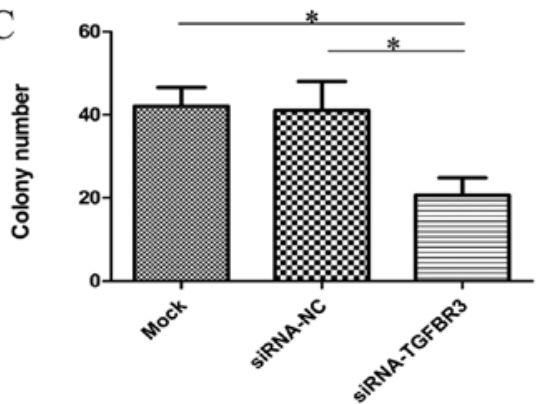

Figure 3. Knockdown of TGFBR3 reduces T24 cell viability. (A) Cell growth curve showed the growth inhibition in the T24 cells with siRNA-TGFBR3 by WST-1 assay. ${ }^{* *} \mathrm{P}<0.01,{ }^{* * *} \mathrm{P}<0.001$. (B) Colony formation ability of siRNA-TGFBR3 cells. Images are representative of colony formation ability of one filed. (C) Average colony number is compared between the siRNA-TGFBR3 group and the mock or siRNA-NC-treated group. "P<0.05. Columns, mean of 3 independent experiments; bars, SD.
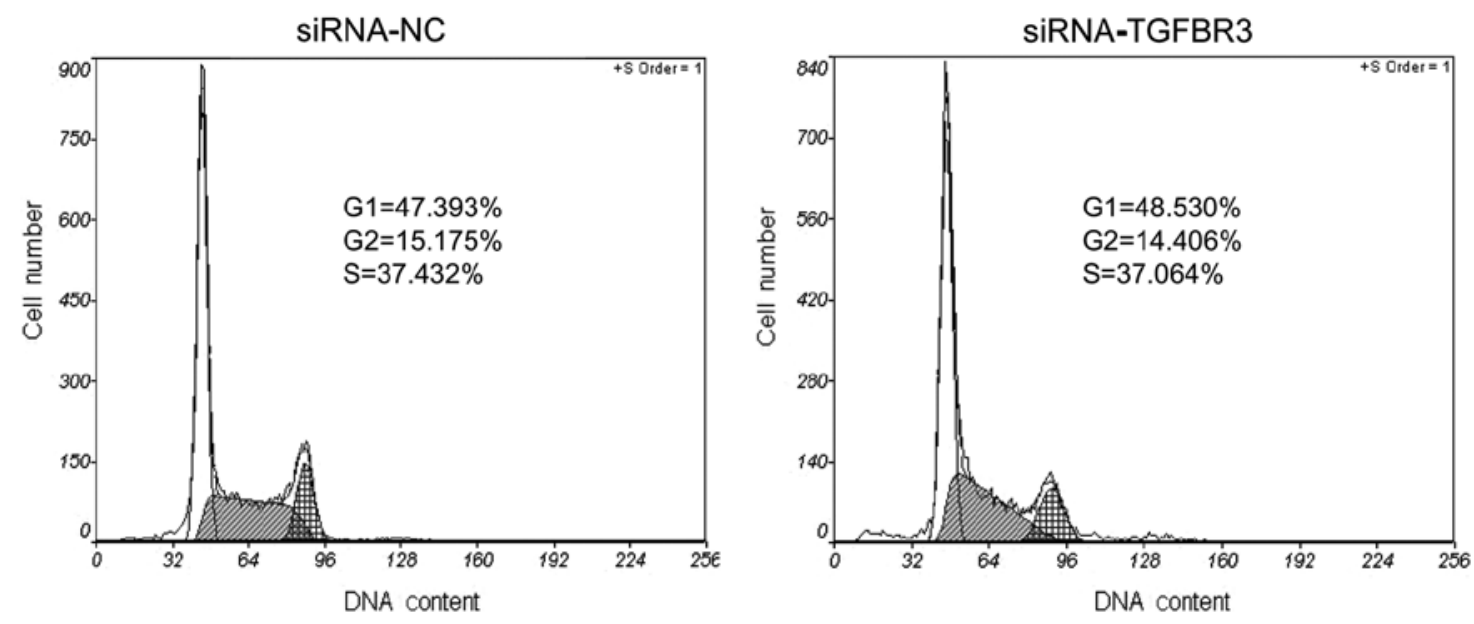

Figure 4. Cell cycle assay in T24 cells by flow cytometric analysis. Proliferating T24 cells were treated with $50 \mathrm{nM}$ siRNA-NC or siRNA-TGFBR3. Forty-eight hours after the treatments, the cells were fixed and stained with PI for the determination of cell cycle distribution. Representative histograms of cell cycle distribution are shown. Data were obtained from 3 independent experiments.

post-transfection, respectively. Compared to the mock and siRNA-NC, TGFBR3 expression in siRNA-TGFBR3 group decreased significantly.

To determine whether TGFBR3 expression affects cell proliferation and growth, we knocked down TGFBR3 in T24 cells and analyzed cell viability using a WST-1 assay. In the experiment, there was no clear difference in cell proliferation among the blank, mock and siRNA-NC groups. The proliferation of cells transfected with siRNA-TGFBR3, however, was notably inhibited from 48 to $72 \mathrm{~h}$ as compared to the siRNANC group (Fig. 3A).

Similarly, the colony formation assay showed that the total colony number of the siRNA-TGFBR3 group was less than that of the siRNA-NC group, and that there was no evident difference in colony formation among the blank and siRNA-NC groups (Fig. 3B and C).

To determine whether the inhibition of the cell viability and colony formation is related to cell cycle arrest, the phase distribution of the cell cycle was analyzed by flow cytometry. Compared to the siRNA-NC group, there was no marked change of cell cycle distribution in the siRNA-TGFBR3 group (Fig. 4).

Since the cell viability is $\sim 2 / 3$ in the siRNA cells at $48 \mathrm{~h}$ (Fig. 3A) and the cell cycle distribution is the same as the control cells (Fig. 4), and the cell death seems to be the same from sub-G1/G0 cell numbers in this figure, the explanation for the low viability is the slow cell growth and the presence of the same cell cycle pattern.

Knockdown of TGFBR3 decreases the migration and invasion of T24 cells. To determine whether TGFR 3 expression is associated with cellular migration and invasion, we detected cell migration ability in TGFR3-knockdown T24 cells using a monolayer wound healing assay and a transwell migration assay. The siRNA-NC-treated cells consistently migrated faster than the siRNA-TGFBR3-treated cells in the monolayer wound healing assay (Fig. 5A), while the transwell migration assay demonstrated a trend toward a bigger increase in the basal migration of siRNA-TGFBR3-treated cells than in the siRNA-NC-treated cells (Fig. 5B and C). 


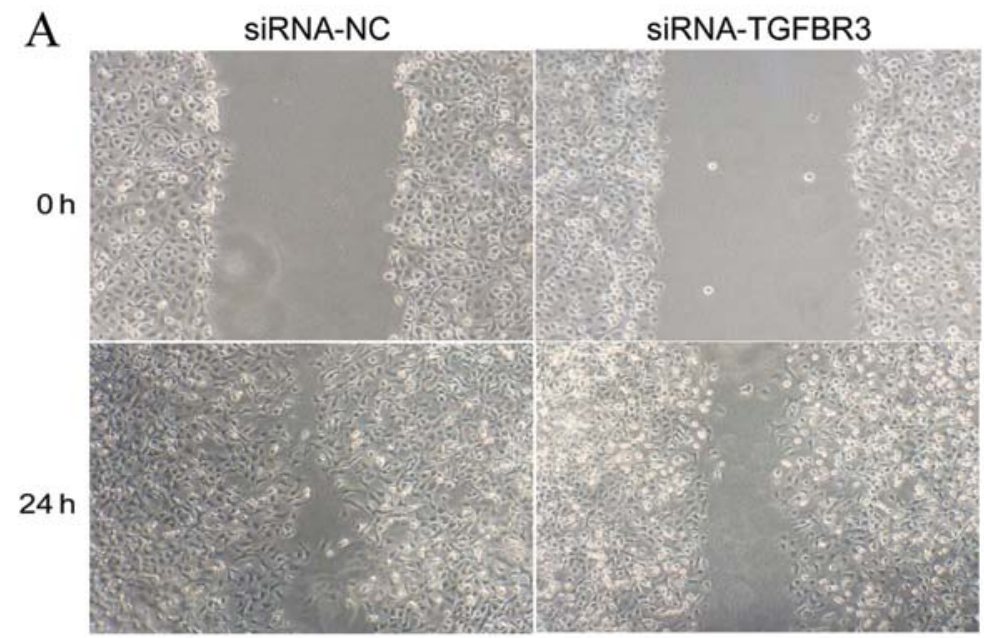

B

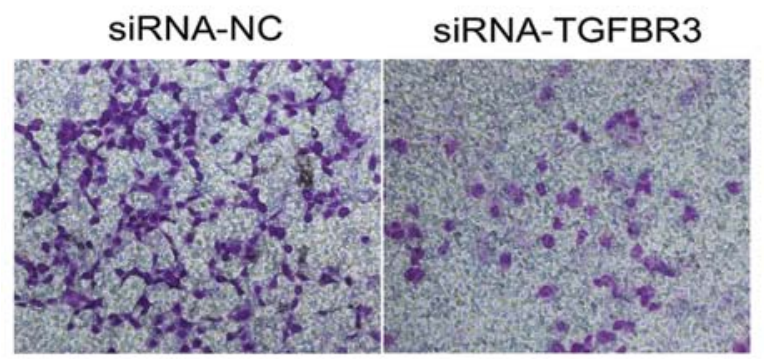

$\mathrm{C}$

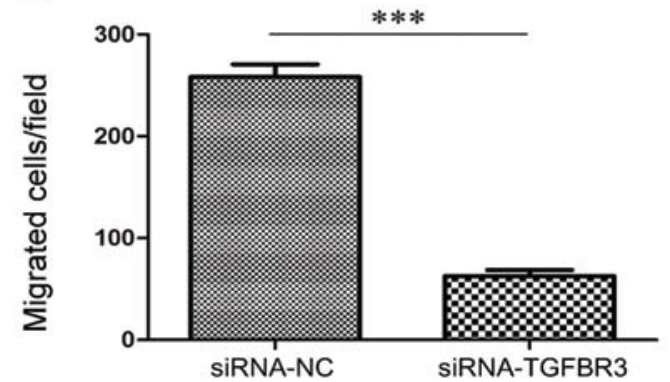

Figure 5. Knockdown of TGFBR3 reduces migration of T24 cells. (A) The cell motility of transfected T24 cells was evaluated using the monolayer wound healing assay. Migration of the cells into the scratch was observed at time-points of 0 and 24 h. Data were obtained from 3 independent experiments. (B) T24 cells transfected with $50 \mathrm{nM}$ siRNA-NC or siRNA-TGFBR3 were seeded in the upper chamber of transwell filters. After a 24-h incubation, the top of the filters was scraped and cells that migrated through the filters were fixed and stained. Images are representative of migrated cells of one filed. (C) The number of migrated cells in the siRNA-TGFBR3 3 group was lower than in the siRNA-NC group. ${ }^{* * *} \mathrm{P}<0.001$. Columns, mean migrated cell number of 3 independent visual fields; bars, SD.

A

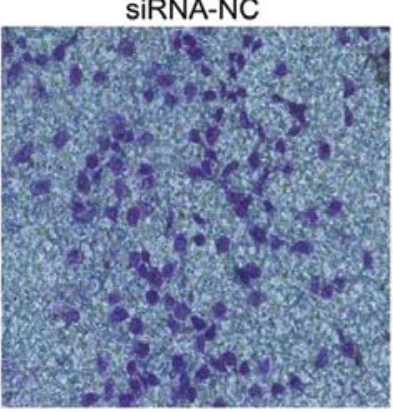

siRNA-TGFBR3

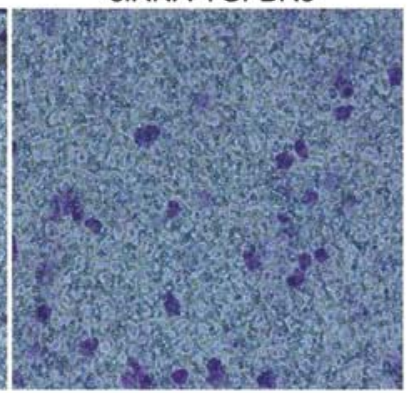

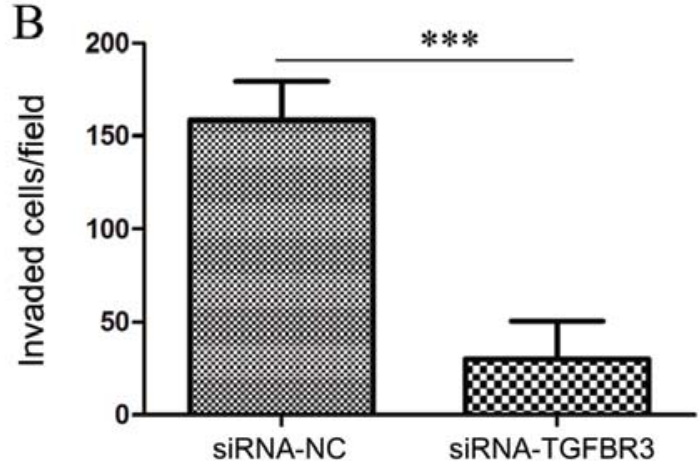

Figure 6. Knockdown of TGFBR3 decreases cell invasion through Matrigel. (A) T24 cells transfected with $50 \mathrm{nM}$ siRNA-NC or siRNA-TGFBR3 were seeded in the upper chamber of transwell filters. After a 24-h incubation, the top of the filters were scraped and cells that Matrigel-invaded through the filters were fixed and stained. Images are representative of invaded cells of one field. (B) The number of invaded cells in the siRNA-TGFBR3 group was lower than in the siRNA-NC group. ${ }^{* * *} \mathrm{P}<0.001$. Columns, mean invaded cell number of 3 independent visual fields; bars, SD.

We used the reconstituted extracellular matrix, Matrigel, to mimic the basement membrane and examined the role of TGFBR 3 on T24 cancer cell invasion in vitro compared with the cells treated with siRNA-NC, the T24 cells treated with siRNA-TGFBR3 significantly inhibited their ability to invade Matrigel (Fig. 6A and B).

\section{Discussion}

Bladder cancer is the most common tumor of the urinary tract. The global age standardised incidence rate is 10.1 per 100,000 males and 2.5 per 100,000 females (16). A report estimated that in 2008, 386,000 cases of the cancer were diagnosed, out 
of which 150,200 patients succumbed to the disease worldwide (17). Therefore, the search for new treatment strategies is urgent.

It has been reported that the loss of TGFBR3 expression directly mitigated the effects of TGFBR3 on regulating cell migration, invasion, proliferation and angiogenesis. This suggests that TGFBR3 acts as a suppressor of cancer progression and/or as a metastasis suppressor, directly influencing breast, prostate, ovarian, pancreatic, renal, non-small cell lung cancer and endometrial carcinoma (7).

In the present study, we identified that TGFBR3 expression was reduced in most superficial BUCs compared to the corresponding normal tissues, while the expression of TGFBR3 typically increased in invasive samples. In addition to identifying TGFBR3 expression in bladder cancer tissues, we also observed TGFBR3 expression in 3 different cells: human normal bladder epithelium cell line SV-HUC-1, superficial derived bladder cancer cell line 5637 and invasive bladder cancer cell line T24. In the present study, too, TGFBR3 expression was suppressed in 5637 cells and heightened in T24 cells. These data are different from previous reports on TGRBR3 and suggest that TGFBR3 may have a different role in BUCs, functioning to suppress tumor progression in early stages but promoting tumor progression during invasion.

In order to further characterize the role of TGFBR3 in invasive bladder cancer cells, we used specific siRNAs to knock down TGFBR3 expression in T24 cells and evaluated the biological effects. We demonstrated that silencing TGFBR3 expression could notably deregulate cell growth, motility and invasion in vitro. This result indicates that TGFBR3 may play a tumor promoter role in invasive bladder cancer cells.

In fact, Criswell et al (18) reported an oncogenic role for TGFBR3 in human and murine breast cancer cells. Knockdown of TGFBR3 resulted in decreased growth, motility and invasion, which are dependent on NF- $\mathrm{B}$ signaling. Recently, Gatza et al (15) reported that TGFBR3 expression is maintained and enhanced in human colon cancer and functions to promote colon cancer progression through the promotion of proliferation, migration, anchorage-independent growth and resistance to apoptosis.

Given that TGF- $\beta$ has numerous and often opposite cellular effects, serving as a tumor promoter and a tumor suppressor, and as an inhibitor and stimulator of cellular proliferation, apoptosis and angiogenesis $(19,20)$, it is possible that, similar to TGF- $\beta$, TGFBR3 also plays different roles in different stages, possibly dependent upon the activation or inhibition of other oncogenes or tumor suppressor genes.

Collectively, in the present study we observed the loss of TGFBR3 expression in superficial bladder cancer, but an increase of TGFBR3 expression in invasive bladder cancer. Furthermore, knockdown of TGFBR3 in invasive bladder cancer cells resulted in decreased growth, motility and invasion. These data revealed that TGFBR3 may act as a suppressor in early stages and as a promoter in later stages of BUCs. We consider that targeting TGFBR3 in the future may become a promising anticancer strategy against bladder cancer. However, further studies are required to determine TGFBR3 status and its potential impact on TGFBR3-based therapy.

\section{Acknowledgements}

The authors acknowledge the participation and cooperation of patients with bladder urothelial carcinoma. The present study was in part supported by grants from the National Natural Science Foundation of China (81171894 to H.T.Z.), the Program for New Century Excellent Talents in University (NCET-09-0165 to H.T.Z.), the '333' Project of Jiangsu Province Government (to H.T.Z.), the Soochow Scholar Project of Soochow University (to H.T.Z), the Suzhou Key Laboratory for Molecular Cancer Genetics (SZS201209 to H.T.Z), the Suzhou Science and Education Youth Health Foundation (KJXW2012014 to X.L.L.) and the Scientific Innovation Research of College Graduate of Jiangsu Province (CXZZ12_0844 to X.L.L.).

\section{References}

1. Fleshner NE, Herr HW, Stewart AK, Murphy GP, Mettlin C and Menck HR: The National Cancer Data Base report on bladder carcinoma. The American College of Surgeons Commission on Cancer and the American Cancer Society. Cancer 78: 1505-1513, 1996.

2. Babjuk M, Oosterlinck W, Sylvester R, Kaasinen E, Bohle A, Palou-Redorta J and Roupret M: EAU guidelines on non-muscleinvasive urothelial carcinoma of the bladder, the 2011 update. Actas Urol Esp 36: 389-402, 2012 (In Spanish).

3. Di Stasi SM, Valenti M, Verri C, Liberati E, Giurioli A, Leprini G, Masedu F, Ricci AR, Micali F and Vespasiani G: Electromotive instillation of mitomycin immediately before transurethral resection for patients with primary urothelial nonmuscle invasive bladder cancer: a randomised controlled trial. Lancet Oncol 12: 871-879, 2011.

4. DeGraff DJ, Clark PE, Cates JM, Yamashita H, Robinson VL, Yu X, Smolkin ME, Chang SS, Cookson MS, Herrick MK, Shariat SF, Steinberg GD, Frierson HF, Wu XR, Theodorescu D and Matusik RJ: Loss of the urothelial differentiation marker FOXA1 is associated with high grade, late stage bladder cancer and increased tumor proliferation. PLoS One 7: e36669, 2012.

5. Kaufman DS, Shipley WU and Feldman AS: Bladder cancer. Lancet 374: 239-249, 2009.

6. Massague J: TGF $\beta$ in cancer. Cell 134: 215-230, 2008.

7. Gatza CE, Oh SY and Blobe GC: Roles for the type III TGF- $\beta$ receptor in human cancer. Cell Signal 22: 1163-1174, 2010.

8. Siegel PM and Massague J: Cytostatic and apoptotic actions of TGF- $\beta$ in homeostasis and cancer. Nat Rev Cancer 3: 807-821, 2003.

9. Dong M, How T, Kirkbride KC, Gordon KJ, Lee JD, Hempel N, Kelly P, Moeller BJ, Marks JR and Blobe GC: The type III TGF- $\beta$ receptor suppresses breast cancer progression. J Clin Invest 117: 206-217, 2007.

10. Turley RS, Finger EC, Hempel N, How T, Fields TA and Blobe GC: The type III transforming growth factor- $\beta$ receptor as a novel tumor suppressor gene in prostate cancer. Cancer Res 67: 1090-1098, 2007.

11. Hempel N, How T, Dong M, Murphy SK, Fields TA and Blobe GC: Loss of betaglycan expression in ovarian cancer: role in motility and invasion. Cancer Res 67: 5231-5238, 2007.

12. Gordon KJ, Dong M, Chislock EM, Fields TA and Blobe GC: Loss of type III transforming growth factor $\beta$ receptor expression increases motility and invasiveness associated with epithelial to mesenchymal transition during pancreatic cancer progression. Carcinogenesis 29: 252-262, 2008.

13. Finger EC, Turley RS, Dong M, How T, Fields TA and Blobe GC: T $\beta$ RIII suppresses non-small cell lung cancer invasiveness and tumorigenicity. Carcinogenesis 29: 528-535, 2008.

14. Cooper SJ, Zou H, Legrand SN, Marlow LA, von Roemeling CA, Radisky DC, Wu KJ, Hempel N, Margulis V, Tun HW, Blobe GC, Wood CG and Copland JA: Loss of type III transforming growth factor- $\beta$ receptor expression is due to methylation silencing of the transcription factor GATA3 in renal cell carcinoma. Oncogene 29: 2905-2915, 2010.

15. Gatza CE, Holtzhausen A, Kirkbride KC, Morton A, Gatza ML, Datto MB and Blobe GC: Type III TGF- $\beta$ receptor enhances colon cancer cell migration and anchorage-independent growth. Neoplasia 13: 758-770, 2011. 
16. Ploeg M, Aben KK and Kiemeney LA: The present and future burden of urinary bladder cancer in the world. World J Urol 27: 289-293, 2009.

17. Jemal A, Bray F, Center MM, Ferlay J, Ward E and Forman D: Global cancer statistics. CA Cancer J Clin 61: 69-90, 2011.

18. Criswell TL, Dumont N, Barnett JV and Arteaga CL: Knockdown of the transforming growth factor- $\beta$ type III receptor impairs motility and invasion of metastatic cancer cells. Cancer Res 68 7304-7312, 2008.
19. Elliott RL and Blobe GC: Role of transforming growth factor $\beta$ in human cancer. J Clin Oncol 23: 2078-2093, 2005.

20. Pardali K and Moustakas A: Actions of TGF- $\beta$ as tumor suppressor and pro-metastatic factor in human cancer. Biochim Biophys Acta 1775: 21-62, 2007. 